\title{
La utilidad lógica como criterio de verdad para determinar la esencia de las cosas en el proceso del conocimiento sensible
}

\section{The utility logic as a criterion of truth to determine the essence of things in the process of the sensitive knowledge}

\author{
Mario Gálvez ${ }^{1}$
}

\section{RESUMEN}

Establecer cómo el cerebro determina la esencia de las cosas es un problema hasta hoy no resuelto, a través de la presente investigación se intenta demostrar que uno de los criterios de verdad que se utilizan en el proceso de conocimiento sensible es la utilidad lógica. Para ello se realizó una investigación en dos fases, la primera de carácter cuantitativa y la segunda cualitativa. La primera fase logró determinar que no basta el uso de los sentidos para determinar la esencia de los objetos, en experimentos visuales realizados a 73 niños se pudo establecer que cerca del 95\% no podía establecer la esencia de los objetos mostrados. Sin embargo, al momento de conocer su utilidad el $90 \%$ podía fácilmente establecer la esencia del mismo objeto. Para la segunda fase se utilizó el método fenomenológico, a través de este, se pudo describir e interpretar el fenómeno investigado. El proceso de investigación permitió concluir que el criterio de verdad que se utiliza para determinar la esencia de las cosas en el proceso del conocimiento sensible es la utilidad lógica inferida gracias a la experiencia acumulada.

Palabras clave: Utilidad lógica, criterio de verdad, conocimiento sensible.

\section{ABSTRACT}

To establish how the brain determines the essence of things is an unresolved problem until nowadays. This research has the intention to demonstrate that one of the truth criterions that are used in the process of sensitive knowledge is the logical utility. Therefore, the investigation was conducted into two phases; the first one of quantitative character and the second of qualitative character. The first phase accomplished to determine that is not enough the use of senses to identify the object essence. In a visual experiment to 73 children, it was possible to stablish that close to $95 \%$ could not confirm the essence of the objects that were exhibited. However, when they know their usefulness almost $90 \%$ could easily confirm the essence of the same objects. The phenomenological method was used for the second phase, through this method you could describe and interpret the investigated phenomenon. The research process allowed reaching the final conclusion that the criterion of truth that is used to determine the essence of thigs in the process of sensitive knowledge is the logical utility inferred due to the accumulated experience.

Key words: Utility logic, criterion of truth, sensitive knowledge 


\section{INTRODUCCIÓN}

¿Qué es el conocimiento? ¿Qué es eso que día a día el sistema educativo se propone como tarea colocar en los cerebros de los estudiantes? Responder que el conocimiento es una construcción no contesta totalmente la pregunta, pues inmediatamente nos saltan las preguntas ¿sobre qué se construye? ¿Cómo se lo construye? No construimos los objetos en nuestra mente, entonces, ¿qué es lo que construimos?

Las investigaciones psicogenéticas que se han hecho durante sesenta o setenta años en la escuela ginebrina nos dicen que lo que se construye es la forma de organizar las interacciones con el mundo externo.

Conocer es organizar los datos de la realidad, darles un sentido, darles una utilidad. El ser humano puede captar un objeto en tres diferentes niveles: el sensible, el conceptual y el holístico. (Castorina, 2012)

No cabe duda que los tres niveles son igualmente importantes, sin embargo, creemos que si no se parte de un buen desarrollo del aprendizaje sensible, los demás tendrán serias limitaciones.

El conocimiento sensible consiste en captar un objeto por medio de los sentidos. Gracias a ellos podemos almacenar en nuestra mente las imágenes de las cosas, con color, figura y dimensiones. Los ojos y los oídos son los principales sentidos utilizados por el ser humano. (Fraenza, Yonahara \& Parié, 2013)

Es durante el proceso del conocimiento sensible donde, lo que hemos denominado Utilidad Lógica puede tomar una importancia significativa, organizando las sensaciones que permiten diferenciar una mesa de una silla.

Estas estructuras lógicas están conectadas por una lógica que le brinda a la aprehensión mental características funcionales para diferenciarlo. Es ahí donde radica nuestra investigación, que tie- ne como objetivo establecer si la Utilidad Lógica es criterio de verdad que usan los niños de cinco años para determinar la escencia de los objetos en el proceso del conocimiento sensible.

Comprobar la importancia de la utilidad lógica en el proceso del conocimiento sensible para utilizarla en modelos de aprendizaje que busquen entregarle al alumno no solo el conocimiento, sino también la razón y utilidad en su enseñanza para que así pueda permanecer en la mente.

\section{Hipótesis:}

La utilidad lógica sería el criterio de verdad que usan los niños de 5 años para determinar la esencia de los objetos en el proceso del conocimiento sensible.

\section{MÉTODOS Y MATERIALES}

La investigación se desarrolló utilizando instrumentos metodológicos idóneos en el estudio cuanti-cualitativo. Para la etapa descriptiva se utilizó una encuesta y para la cualitativa se usó la fenomenología, donde se indagó sobre cuestiones de significado, esta consistió en la triangulación de técnicas para la recolección, las cuales fueron: la observación, la entrevista y las anotaciones en bitácora.

El fenómeno fue interpretado usando el método fenomenológico de Spiegelberg (Soto Ramirez, 2013), para demostrar que la utilidad lógica de los objetos es el criterio de verdad que ser humano utiliza para determinar la esencia de los objetos.

La muestra estuvo constituida por un total de 73 niños de 5 años distribuidos en 5 grupos. Para la etapa descriptiva se realizó la investigación con el total de niños (censo), mientras que para la etapa cualitativa, de diseño fenomenológico, se utilizó las muestras de caso-tipo, que de acuerdo al método fenomenológico, fue incrementándose hasta que los caso-tipo dejaron de brindar nuevos datos a la investigación. Las etapas fueron las siguientes: 
Para la parte cuantitativa. Se mostró a los alumnos 2 gráficos con 2 objetos sin ser utilizados, para saber, a través de una encuesta, si eran capaces de determinar la esencia de dichos objetos. Luego se les presentó nuevamente los gráficos, esta vez con personas haciendo uso de ellos. Seguidamente se volvió a encuestar para saber si el uso de los objetos permitía que los alumnos determinaran su esencia.
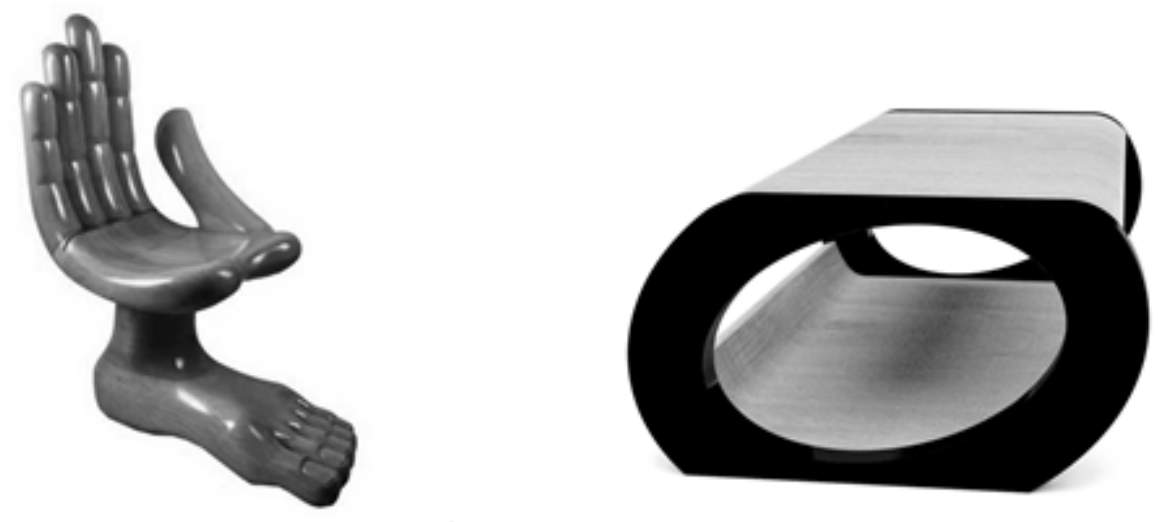

Figura $N^{\circ}$ 1. Gráficos de objetos sin ser utilizados.
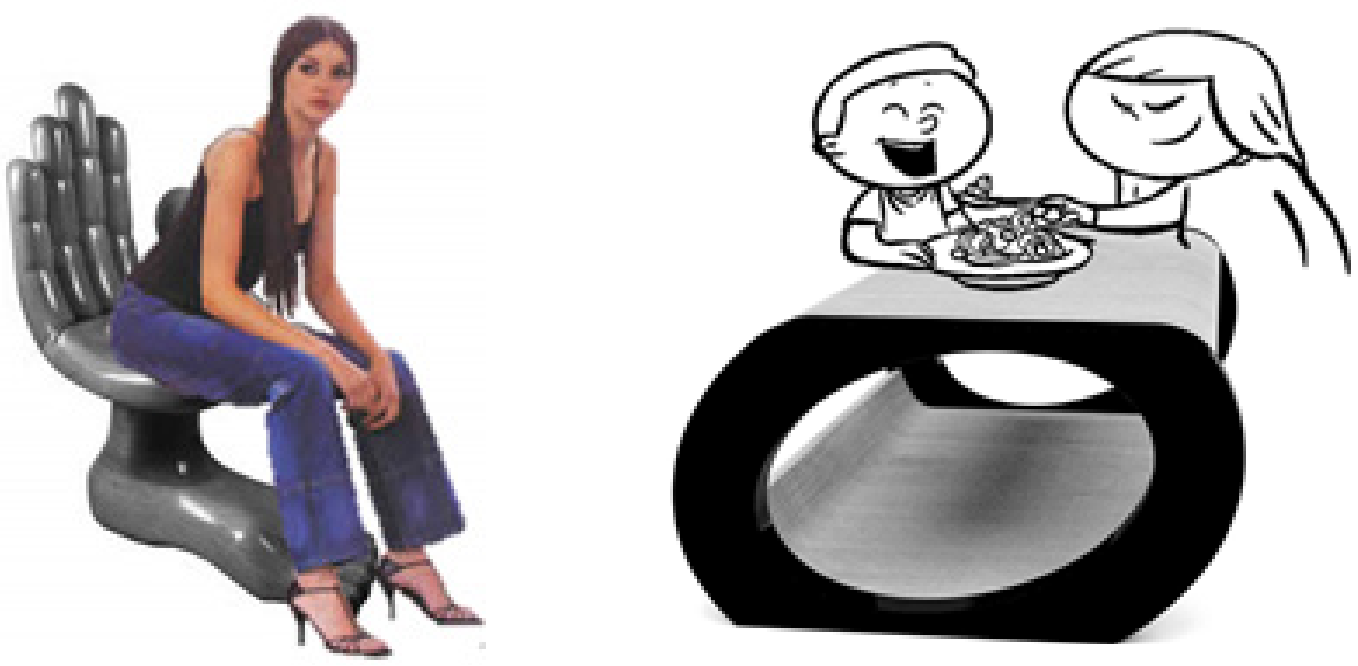

Figura $\mathrm{N}^{\circ}$ 2. Gráficos de objetos utilizados.

Para la parte cualitativa. Esta se realizó de la siguiente manera:

a. Entrega de objetos a los niños. Se le mostró al niño dos objetos que desconocía, en este caso un masajeador de mano y una brújula pero sin mencionarles la utilidad de los objetos para establecer si podían determinar su esencia.
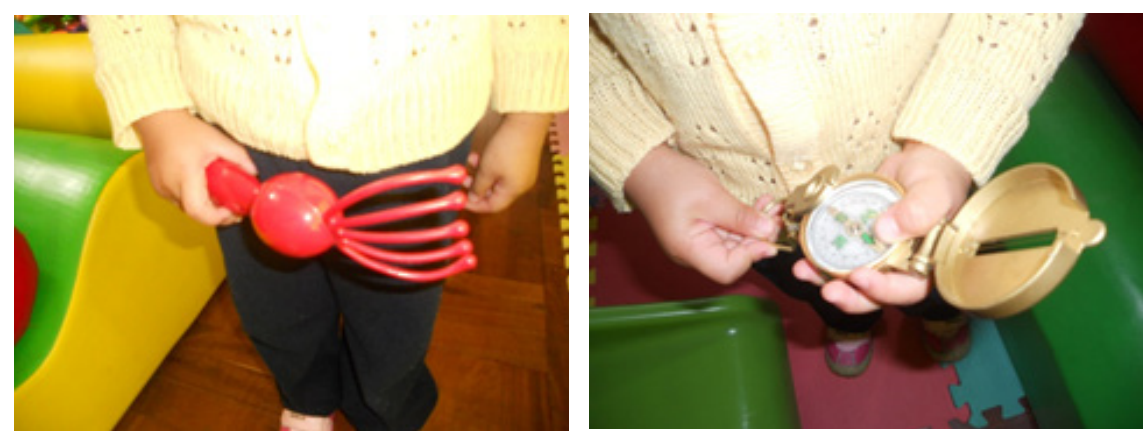

Figura $\mathrm{N}^{\circ}$ 3. Masajeador y brújula mostrada a los niños. 
b. Primera observación. Se observó las acciones que realizaban los niños con los objetos entregados y se anotó en la bitácora

c. Primera ronda de entrevistas. Se entrevistó a los niños, a los padres y a los maestros para analizar sus puntos de vista sobre los resultados de la primera observación.

d. Mostrar la utilidad de los objetos. Se mostró a la mitad de los niños una forma de utilizar los objetos, mientras que a la otra mitad se le mostró una utilidad distinta.

e. Segunda observación. Se observó las acciones que realizaban los niños con los objetos a los que se les había mostrado su utilidad y se anotó en la bitácora.

f. Segunda ronda de entrevistas. Se entrevistó a los niños, a los padres y a los maestros para analizar sus puntos de vista sobre los resultados de la segunda observación ahora que los niños conocían la utilidad de los objetos mostrados.

g. Solicitar objetos según la utilidad mostrada. Se pidió a los niños que reconocieran los objetos de acuerdo a la utilidad mostrada y se anotó las observaciones en la bitácora.

h. Tercera ronda de entrevistas. Se entrevistó a los niños, a los padres y a los maestros para analizar sus puntos de vista sobre los resultados de lo observado.

\section{RESULTADOS}

La investigación demostró que, en los niños, la esencia de los objetos mostrados cambia. Este cambio de esencia no se produce por la modificación de las características perceptibles de los objetos, sino por el cambio de utilidad que tiene el objeto en su conjunto.

Como sabemos, las características percibidas de un objeto no se alojan en un solo sitio de nuestro cerebro (Mora, 2011), como ejemplo, tan solo las características percibidas de la visión van a nueve regiones distintas del cerebro para poder comprender lo que vemos. Sin embargo, increíblemente nuestro cerebro es capaz de determinar la esencia de los objetos ligando los cientos de características que perciben todos nuestros sentidos.

Ligar estas características es una habilidad propia de los cerebros que, gracias a su evolución, han podido razonar. (Maturana, 1992). Y en el caso de los objetos sensibles, la razón necesita de un criterio con el cual pueda ligar las características percibidas y así poder inferir la esencia del objeto para asignarle una categoría de acuerdo a las abstracciones que, producto de la experiencia, se han concebido en el cerebro. (Piaget, 2010). Este criterio, llamado criterio de verdad, debe concordar con la realidad inmediata del objeto percibido (Hesse, 1951).

Para que pueda concordar con el objeto real es insuficiente, para la razón, las características percibidas del objeto. Este necesita, indispensablemente, el conocer la utilidad del objeto percibido, ya sea informandose a través de la inferencia de experiencias para poder determinar su esencia.

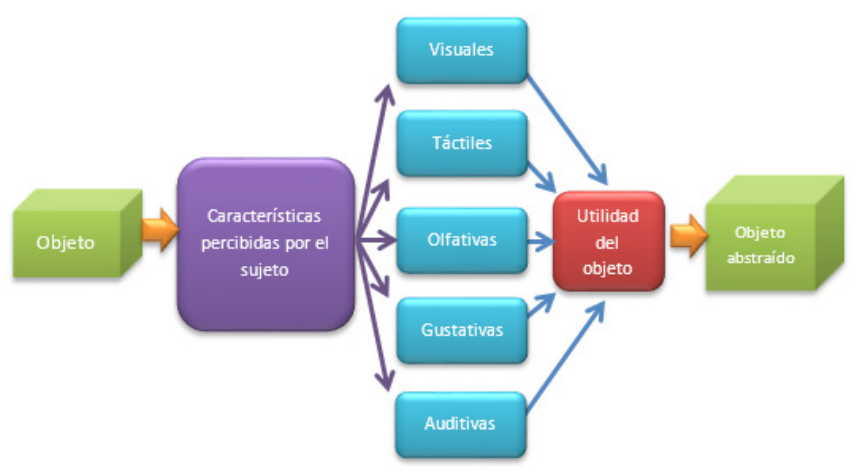

Figura $\mathrm{N}^{\circ} 4$

Si esta utilidad cambia, entonces se modificará también la esencia del objeto a pesar de que las características percibidas no se alteran.

Una vez conocido esta utilidad, la razón busca de entre sus experiencias previas para asignarle al objeto una esencia, este proceso es una predic- 
ción lógica (Piscoya Hermoza, 1995) que sigue la siguiente fórmula:

Premisa I: Para todo objeto real " $x$ ", si “ $x$ " tiene la utilidad real de "P", entonces " $x$ " tiene la esencia real de "Q"

Premisa II: "a" tiene la utilidad real de "P"

Por lo tanto, Premisa III: "a” tiene la esencia real de "Q"

\section{DISCUSIÓN}

\section{Discusión sobre la hipótesis:}

La hipótesis planteada en la investigación es: “La utilidad lógica es el criterio de verdad que usan los niños de 5 años para determinar la esencia de los objetos en el proceso del conocimiento sensible."

La investigación ha permitido aceptar la hipótesis planteada, pues los resultados pudieron determinar que si a un objeto se le modificaba su utilidad real, también se le modificaba su esencia. Este cambio en su esencia se producía a pesar de que las características reales perceptibles del objeto permanecían intactas.

Por esta razón, no basta la percepción para que los niños de 5 años puedan determinar la esencia de los objetos, es necesario que las características percibidas e interpretadas individualmente en el cerebro se liguen a través de un criterio de verdad.

Este criterio, según la presente investigación, es la utilidad lógica la cual sigue la fórmula lógica ya descrita en los resultados

\section{Discusión sobre los antecedentes de la investiga- ción:}

Asimismo, la investigación realizada respalda la investigación de los científicos del Instituto de
Tecnología de Massachusetts, quienes al resolver el "problema de Molyneux" en abril del 2011, determinaron que el cerebro representa objetos en la mente dependiendo del sentido con el que se ha percibido y es interpretada por la experiencia del sujeto.

Por ese motivo, los niños de la presente investigación no pudieron determinar la esencia de los objetos pues no tenían las suficientes experiencias en la mente para relacionarlas con las percepciones que estaban viviendo.

\section{CONCLUSIONES}

1. La utilidad lógica es el criterio de verdad que usan los niños de la sección de 5 años para determinar la esencia de los objetos en el proceso del conocimiento sensible.

2. No es posible que los niños de 5 años puedan determinar la esencia de los objetos solo con la percepción.

3. La utilidad de un objeto es una información que los niños de 5 años necesitan para determinar su esencia en el proceso del conocimiento sensible.

4. El cambio de utilidad lógica de un objeto provoca un cambio en la esencia del mismo objeto por parte de los niños de 5 años.

\section{REFERENCIAS BIBLIOGRÁFICAS}

Ariely, D., 2013. Las trampas del deseo. 4ta. ed. Barcelona: Editorial Planeta S.A. pp. 132

Castorina,J.A, (2012), Psicología y Epistemología Genética. Buenos Aires: Lugar editorial.

Fraenza, F; Yonohara, S \& Parié, A. (2013), ¿Cómo vemos?. Córdova, Editorial Brujas.

Hesse, J., 1951. Teoría del Conocimiento. Buenos Aires: Editorial Contemporánea. pp. 129-134

Maturana, H. y. V. F., 1992. El árbol del conocimiento. Santiago de Chile: Editorial Universitaria. pp. 97-106 
Mora, F., 2011. ¿Cómo funciona el cerebro? Madrid: Editorial Alianza. p. 101

Piaget, J., 2010. La psicología de la inteligencia. Barcelona: Editorial Crítica. pp. 64-93

Pinillos, J. L., 2001. La mente humana. Madrid: Ediciones Temas de Hoy. pp. 85-96

Piscoya Hermoza, L., 1995. Investigación científica y educacional. Segunda Edición ed. Lima: Editorial Amaru Editores. pp. 149-162

Soto Ramirez, R., 2013. Investigación cualitativa. [En línea] Available at: www.paginaspersonales.unam.mx/files/981/ CURSO2013ok.pdf [Último acceso: 26 agosto 2015]. 